\title{
Observaciones sobre la representación sintáctica de los determinantes
}

\author{
JOAQUIN MESA \\ Universidad de Córdoba
}

0. Conceptos como los de núcleo, elemento obligatorio, centro y sus correspondientes, elementos opcionales, o complementos y determinantes..., pese a su frecuencia de uso en los distintos modelos gramaticales, sufren, como otros muchos conceptos sintácticos, de una vaguedad e imprecisión que dificultan, en ocasiones, la comparación entre distintos análisis. Los intentos de delimitación más recientes muestran la complejidad y dificultad de conceptos tan frecuentemente utilizados y de aparente simplicidad. Prueba de ello es que los criterios que para la determinación del elemento nuclear usa el, ya clásico, estudio de A. Zwicky (1985) son discutidos por R. Hudson (1987), de modo que se defiende la nuclearidad de elementos, para A. Zwicy, complementarios.

Es nuestro propósito en las páginas que siguen realizar algunas observaciones sobre estos conceptos, su validez y uso, para una frase nominal. Los problemas que estos conceptos presentan para una estructura de frase verbal son algo distintos y se entrelazan con complejas relaciones sintáctico-semánticas como las de transitividad, ergatividad, complementos circunstanciales...

Por ello, nuestras conclusiones se refieren exclusivamente a la frase nominal. Su posible validez para el análisis de una frase verbal merecerian estudio aparte. Sin embargo, creemos que en las recientes discusiones sobre el complemento circunstancial- en el sentido de G. Rojo- o en los distintos tipos de suplemento establecidos recientemente por E. Alarcos subyace el concepto de núcleo, elemento obligatorio, elemento subcategorizado, y complemento de la frase verbal; la no posibilidad de conmutar por cero un elemento considerado inicialmente marginal motiva la consideración de un nuevo tipo de complemento: "Circunstancial", "aditamento circunstancial", o "pseudo-circunstanciales" (BrucartHernanz 1987:271 y ss.).

1. Muy brevemente expondremos algunas observaciones teóricas sobre el concepto de núcleo, cuestiones surgidas a partir de los trabajos más recientes sobre el tema.

Parece ser la Tagmémica el primer modelo lingüistico que prestó particular antención al concepto de núcleo (González Escribano 1979-80), ya que con anterioridad este con- 
cepto no tenia, ni aun hoy, unos limites claros, a pesar de su centralidad para una teoría de la estructura de frase.

La Tagmémica llegó a manejar hasta 16 criterios- en distintas combinaciones, no simultáneamente- de nuclearidad (González Escribano 1979-80:270-282): independencia, tamaño de la clase, versatilidad, endocentricidad, frecuencia, prominencia prosódica, longitud, prominencia semántica, coherencia de análisis, valor diagnóstico, obligatoriedad, cohesión, vinculación al predicado, presencia de marcadores, sensibilidad ante las transformaciones e implicación semántica. Y en esta lista, donde se mezclan rasgos y criterios heuristicos, se encuentran, probablemente, los instrumentos, mejor o peor analizados, que se han usado para determinar el concepto de núcleo. Así, por ejemplo, A. Zwicky (1985) maneja cinco: argumento semántico/funtor semántico, próximo al de prominencia semántica; subcategorizador/subcategorizado, cercano, en parte, al de vinculación al predicado; lugar morfosintáctico, rector/regido y marcador de concordancia, que corresponderian aproximadamente a presencia de marcadores, y junto a éstos, los tests sintácticos habituales de endocentricidad y obligatoriedad, que también recogen los tagmemicistas.

Alguno de los rasgos de la Tagmémica- vinculación al predicado - tendría sólo validez para determinar la estructura de predicados, objetivo que de igual modo persigue J.G. Escribano en su trabajo. Puede verse en éste una crítica detallada de cada uno de estos criterios. A nosotros nos interesa poner de relieve algunos aspectos de la investigación sobre el núcleo que nos parecen fundamentales en cualquier discusión sobre este concepto.

1.1. En primer lugar, la necesidad de distinguir los criterios operativos o test de las caracteristicas inherentes del elemento nuclear. De acuerdo con A.Zwicky (1985:11 y ss.), son dos los tests sintácticos normalmente usados: la equivalencia distribucional, o endocentrismo, y el elemento obligatorio; tests que, en ocasiones, se han considerado rasgos caracteristicos.

1.1.1. Respecto del primero de los criterios operativos, la endocentricidad, A. Zwicky (1985:12) se ve obligado a matizar la definición con un significativo "roughly" respecto de la idéntica distribución de constituyente nuclear y constructo. Del mismo modo, G.Rojo y T.Jiménez (1989:100-1) añaden el requisito de equifuncionalidad para construcción y constituyente central de una relación endocéntrica. La razón de estas modificaciones es manifiesta: la insuficiencia del concepto de posición sintáctica. Incluso con estas modificaciones el criterio no parece poder aplicarse con facilidad. A. Zwicky (1985:12) mantiene que en la frase que a nosotros nos interesa Det $+\mathrm{N}$, $\mathrm{N}$ es el equivalente distribucional de la frase, aunque en nota remite a la opinión contraria de J. Lyons. Tampoco G.Rojo y T. Jiménez (1989) consideran que el nombre es el equivalente distribucional-funcional de la frase (vid. 2.2).

1.1.2. La obligatoriedad del constituyente que se considera núcleo tampoco es criterio libre de discusión. La principal cuestión que se plantea es la siguiente: ¿ qué sucede en el caso de que el elemento nuclear, y por ello obligatorio, de la construcción no aparezca? Parece que se ofrecen dos posibilidades: Debe postularse que el elemento propuesto, por no aparecer obligatoriamente en todos los contextos, no es núcleo-obligatoriedad, de J. 
G.Escribano ( 294 y ss.) y para este autor, concepto no operativo, dado que no habria elementos nucleares en todos los contextos- o bien que el núcleo, por pertenecer a un nivel más alto de abstracción, puede no estar fonéticamente presente en la cadena pero ser elemento obligatorio de la estructura, implicado gramaticalmente- obligatorio ${ }_{2}$ de J. G. Escribano.

Según esta última propuesta, el núcleo seria elemento elidido pero no opcional, dado que debe ser recuperado obligatoriamente por el intérprete (Zwicky: 13) o bien no se trataría de elipsis, sino de que, en ciertos contextos, su exponente es cero fonético (G.Escribano: 300).

En esta cuestión de los elementos vacíos subyacen dos interrogantes. Por un lado, si la no aparición en la cadena no es motivo suficiente para negar carácter nuclear a un constituyente, ¿de qué manera determinamos el elemento nuclear? Y de otro modo, si marginamos en determinados casos pruebas empíricas, ¿en qué nivel de abstracción debemos detenernos? Es posible que, en ocasiones, el sacrificio de pruebas empíricas -asi pensamos en el caso de la frase nominal- no aporte beneficios para la generalidad de la explicación. En suma, son cuestiones que debería responder la teoria del modelo sintáctico de que se trate.

Por otro lado, se plantea la relación entre núcleo y discurso. Parece claro que en contextos adecuados cualquier elemento, nuclear u opcional, puede no estar presente. La explicación de la mayoria de los gramáticos consiste en defender que el elemento nuclear es recuperado necesariamente por el oyente a fin de lograr una interpretación adecuada del mensaje, mientras que en el caso de los elementos opcionales el oyente no precisa recuperar esa información que no aparece, información que no se considera elipsis sino "elisión" O "no realización" (Jiménez Juliá 1991:224 y ss.).

Normalmente, también se postula que la recuperación de información- en el caso de la elipsis frente a otros casos de elementos no explícitos- se produce en marcos textuales, esto es, en el modelo de elipsis habitual se presenta ésta como la recuperación de un elemento nuclear, estructuralmente necesario, que en un enunciado no aparece, pero que aparecía en el enunciado inmediatamente anterior o posterior. Frente a este tipo de accesibilidad de la información, la "elisión" (Jiménez Juliá: ibidem) u obligatorio ${ }_{3}$ de J.G. Escribano se enmarca en ámbitos culturales o situacionales, no textuales.

Estas explicaciones se apoyan en un modelo sintáctico que tiene como límite la oración. La Lingüistica del texto y, especialmente, la Pragmática han mostrado que los procedimientos de recuperación de información por el oyente no están tan claramente delimitados como pretenden estos gramáticos. Por otra parte, se trabaja con un modelo de código en la interpretación de los enunciados, esto es, la tarea del oyente consiste en decodificar la información previamente codificada por el emisor. D. Sperber y D. Wilson (1986: 9 y ss.) mantienen que ese modelo de esquema comunicativo es insuficiente, y que debe ser complementado con el modelo inferencial, esto es, la información que el emisor dispone para el receptor es "completada" por el oyente. De acuerdo con este modelo comunicativo, cualquier secuencia está "incompleta" o "truncada".

En fin, la elipsis es un instrumento en la explicación sintáctica que debería usarse con suma precaución. Los fenómenos de elipsis, elisión o no realización desbordan, a menudo, el límite de explicación de la Sintaxis y la Semántica. Los mecanismos de interpretación son, como sabemos por la Pragmática, complejos. 
1.2. En segundo lugar, surge una cuestión básica sobre los rasgos: ¿el haz de rasgos que se maneja, sin duda heterogéneo y diverso, puede ser subsumido bajo una misma etiqueta conceptual, la de núcleo? Y esta cuestión, ciertamente, apunta a la necesidad y operatividad del concepto de núcleo en sintaxis. Las respuestas son diversas: A. Zwicky parece postular la escasa operatividad de este concepto', mientras que R. Hudson (1987:111) mantiene que permite justamente establecer generalizaciones sobre rasgos y reglas que no serian posibles sin tal concepto.

También se ha definido el núcleo según su posición en la estructura de frase. $R$. Jackendoff (1977:30) vacilaba entre considerar núcleo al elemento de la estructura de frase $\mathrm{X}^{\mathrm{n}-1}$, esto es, cualquier nudo inferior a la proyección máxima, o bien la categoría léxica $X$ que ocupa el lugar más bajo de la estructura y determina las características de la proyección máxima, la frase.

La primera opción tenia la ventaja de ser neutra y vacía de contenido. En una estructura de frase existían tantos núcleos como niveles de proyección. Esta opción, sin embargo, parece descartada implícitamente por el trabajo de R. Jackendoff y explícitamente por S. Rothstein (1989:98). En la segunđa opción, es núcleo la categoria léxica que caracteriza la frase, una definición muy próxima al endocentrismo o "centrality" de A.Kornai y G.K.Pullum (1990:32).

En cualquier caso, lo que nos parece claro es la centralidad del concepto de núcleo en cualquier trabajo sobre Sintaxis. Uno de los propósitos de cualquier modelo sintáctico es poner de relieve las relaciones de constitución y/o dependencia entre los elementos de una cadena. Pues bien, estas relaciones de constitución y dependencia en la mayoria de los casos se expresan de modo jerárquico. Por ello, si se establecen jerarquias, parece necesario determinar cuál o cuáles de los elementos del esquema sintáctico rige, gobierna, determina... a cualesquiere otro. Ciertamente, este carácter central del concepto no ha motivado, como estamos exponiendo, demasiados estudios dedicados al esclarecimiento de un instrumento intuitivo, situación que, por otro lado, no es exclusiva de este concepto básico ni excesivamente anómala en los estudios sobre Sintaxis.

\section{Ante un enunciado ${ }^{2}$ como:}

1. (...) pero él tiene una plena confianza que aquel equipo de médicos es un equipo preparado(..)(p.166)

Las posibilidades de interpretación y representación sintáctica del elemento subrayado

\footnotetext{
' Pese a que, como deciamos, el trabajo citado de A. Zwicky se ha convertido en un clásico para el concepto de núcleo al exponer una serie de ideas y conceptos que raramente se hacian explicitos en los trabajos sintácticos, el propósito de A. Zwicky no era principalmente éste, sino someter a revisión el mal uso, en su opinión, que del concepto de núcleo realizaban algunos estudios de morfología. Sin embargo, la revisión del concepto en morfología le lleva a un examen detenido del concepto en sintaxis, de modo que la concurrencia de varios criterios conduce a soluciones divergentes en la evaluación de los constituyentes y por ello, en su opinión, el concepto no es operativo.

${ }^{2}$ Los ejemplos usados proceden de la obra El habla de la ciudad de Madrid (1981). Debo agradecer a los editores de la misma, los profesores M. Esgueva y M. Cantarero, su generosidad por permitir la reproducción de su trabajo. Asimismo, hago extensivo este agradecimiento al profesor G, Rojo que, muy amablemente, me cedió en diskette este mismo texto.
} 
se reducen, por pura lógica, a tres:

A. el elemento subrayado está en una relación de determinación con respecto al nombre que le sigue, esto es, éste último es el núcleo

B. entre el elemento subrayado y el nombre la relación no es endocéntrica sino exocéntrica, y por ende, no existe núcleo. La relación es de interdependencia.

C. el elemento subrayado es el núcleo, de modo que el nombre desempeña una relación de complementación. Solución divergente a la primera, pero coincide en que se postula una relación de determinación.

La representación sintáctica de la frase nominal del ejemplo será, pues, como sigue:
A) (FN (Det)(N))
B) (FN (Det)(Nominal))
C) (FDet (Det $(\operatorname{Det})[(N))]$

2.1. La primera de las opciones es, probablemente, la opción que cuenta con mayor tradición y que ha sido unánimemente aceptada, hasta muy recientemente, por distintos modelos gramaticales.

A pesar de que los argumentos que apoyan este análisis no se hacen explícitos, parece que podemos rastrear los apoyos que sustentan esta interpretación sintáctica transmitida desde la primera Sintaxis occidental.

Ciertamente, Apolonio Díscolo (1987) no habla de frases y de relaciones nucleares entre los elementos constitutivos de éstas, pero, al comienzo de su Sintaxis, explica que existe un orden, una relación jerárquica entre los elementos de la oración: "El orden de las partes de la oración es una imitación de la oración perfecta, que muy justamente coloca en primer lugar el nombre, después el verbo, puesto que sin ellos ninguna oración queda cerrada(...), y si se le quita el nombre o el verbo la oración no estará completa, pero si se le suprimen todas las demás de ningún modo queda defectuosa" (I,\#12:81).

Maneja Apolonio un concepto de nuclearidad oracional que se sustenta en la sustitución, procedimiento semejante al de independencia de los tagmemicistas- frecuentemente usado, de otro lado, en las gramáticas modernas- y la obligatoriedad. Es elemento obligatorio y por ende, más alto en la jerarquía y orden, aquel que no puede ser suprimido sin modificar la oración perfecta o completa. Esta sintaxis no usa un nivel de análisis intermedio entre cláusula y palabra, de modo que lo que vale para la cláusula es válido para las palabras que la constituyen. De este modo, nombre y verbo son los dos elementos centrales en torno a los cuales se estructuran, como elementos dependientes, las restantes partes de la oración. Esta vieja idea, la obligatoriedad de nombre y verbo para constituir una proposición, procede del análisis lógico, una prueba más de su dominio sobre la gramática.

A. Bello (1847) usa un concepto típico de frase endocéntrica: "Un sustantivo con las modificaciones que lo especifican o explican forma una frase sustantiva, a la cual es aplicable todo lo que se dice del sustantivo; de la misma manera, un verbo con sus respectivas modificaciones forma una frase verbal; (...)(También para adjetivos y adver- 
bios)" (\#81: 191). Se apoya asimismo en la obligatoriedad de sustantivo y verbo en la oración para determinar el núcleo de la frase sustantiva: "La palabra dominante en la oración es el sustantivo sujeto, a que se refiere el verbo atribuyéndole alguna cualidad, acción, ser o estado. Y en torno al sustantivo sujeto o al verbo se colocan todas las otras palabras, las cuales, explicándose o especificándose unas a otras, miran, como a sus peculiares últimos puntos de relación, las unas al sustantivo sujeto, las otras al verbo" (\#478:377).

De forma algo más explícita el texto citado de Bello muestra cómo se produce la derivación de estructura proposicional a estructura de frase: por medio de una generalización inadecuada- el nombre funciona prototípicamente en la clásula como sujeto- se predica del sustantivo su obligatoriedad, nuclearidad, en la frase sustantiva, como la denomina Bello.

El razonamiento parece ser el siguiente: dado que el sustantivo o nombre es elemento obligatorio en una proposición, en la frase en la que se incluye, caracterizada como endocéntrica, desempeñará la función de núcleo. En sintesis, de la obligatoriedad de la función sujeto, prototipicamente desempeñada por un sustantivo, se deduce la nuclearidad del sustantivo en la frase sustantiva. Se pasa inadvertidamente de una relación interfrástica a una relación intrafrástica.

Y no se piense que este tipo de argumentación ha sido ya rechazado por las gramáticas modernas. Una gramática inglesa reciente, la de R. Huddleston (1984:122), considera que adjetivo, nombre y verbo son "major parts of speech", pero nombre y verbo de "special status", porque todas las oraciones contienen al menos un verbo y un nombre; por la mayor complejidad estructural de las frases de las que son núcleos y por último, son más básicos que el resto de las partes de la oración al ser definidos independientemente; y ello, a pesar de que posteriormente y en contradicción con la primera de sus pruebas, afirme que "it is the former (el verbo) that functions as ultimate head of the clause, (...) we can accordingly think of the verb as having the highest ranking in importance in the organisation of the grammar(...) $m^{3}$ (Ibidem). No es de extranar, pues, que en el capítulo

${ }^{s} \mathrm{R}$. Huddleston se apoya curiosamente en un argumento etimológico para sustentar esta última visión del verbo como elemento primordial: " a view reflected in the etymology of the term 'verb' which derives from the Latin for "word" (Ibidem). Asimismo, nuestro primer gramático hizo uso de este argumento para el español: "E llámase verbo, que en castellano quiere dezir palabra, no por que las otras partes de la oración no sean palabras, mas por que las otras sin ésta no hazen sentencia alguna, êsta, por ezcelencia, llamóse palabra" (Nebrija 1992: 184).

Decía más arriba "curiosamente" porque Apolonio argumenta con bases etimológicas para defender to contrario, la precedencia del nombre: "De ahi que prevaleciese la denominación de ónoma (nombre y palabra) para ser aplicada a todas las partes de la oración, debido a su precedencia sobre las demás" (1, \#18: 82). Parece, en cualquier caso, de poco peso la base etimológica para determinar jerarquias y prioridades.

Una coincidencia asimismo sorprendente entre R. Huddleston y Apolonio es la manifestación explícitaimplicitamente, la mayor parte de las gramáticas hacen corresponder la jerarquía al plan del libro- de que esta visión jerárquica de las partes de la oración justifica el orden en el tratamiento y estudio de las mismas, esto es, el plan, el indice del libro, el orden de sus capitulos: "This ranking of the parts of speech(...), provides the basis for the organisation of the descriptive chapters of the book(...) (Huddleston 1984:122), y Apolonio justifica largamente $(1, \# 12-29)$ el orden jerárquico y por ello, la disposición de los capitulos de su libro. 
dedicado a la frase nominal se conceda al nombre la función de núcleo (Huddleston 1984:227).

Y este tipo de argumentación se mantiene incluso cuando se postula una estructura de la cláusula diferente. Pese a defender la endocentricidad de la claúsula y el carácter nuclear de una categoria no léxica sino funcional- la inflexión verbal-, la estructura de constituyentes de la frase nominal no se modifica. Continúa siendo el nombre el elemento nuclear de esta estructura (Haegemann 1991: 89). Parece, pues, evidente que la derivación de la estructura clausal a la estructura de frase no se ha mantenido. No obstante negar el carácter obligatorio al nombre en función de sujeto de la cláusula, el análisis de éste como núcleo de una estructura de frase se conserva.

En suma, este análisis poco explicito deriva inicialmente la nuclearidad del nombre en la frase nominal de la obligatoriedad de éste en la estructura oracional.

Como veremos, la reinterpretación de la estructura de la cláusula también ha modificado en la teoria generativista el análisis de la frase nominal. Pero incluso cuando este análisis de la cláusula se modifica, el análisis de la frase nominal endocéntrica cuyo núcleo es el nombre se mantiene. Sin embargo, a pesar de este cambio en el análisis de la cláusula, no se hacen expresos los motivos de la conservación en el caso de la frase nominal y ello, bien porque no se consideró esta derivación entre cláusula y frase o bien porque se estimó que los argumentos que apoyaban este análisis de nombre como núcleo podian mantenerse. En cualquier caso, sorprende que estos argumentos no se hagan manifiestos.

Aun el escaso grado de explicitud de este análisis, podemos determinar, con relativa seguridad, los argumentos en que se apoya:

A) De acuerdo a lo que más arriba se ha expuesto, la prueba usada explícita o implicitamente para considerar nuclear al sustantivo en una frase nominal deriva de la relación que se establece entre sujeto y predicado, relación en que ambos miembros se consideran obligatorios. Como hemos visto, en alguna ocasión se estima más preeminente el elemento nominal- caso de Apolonio o Bello-, o por el contrario, el elemento verbal( $R$. Huddleston) o bien no se considera nuclear en la oración ni a sujeto ni a predicado, pero se mantiene la nuclearidad del nombre en la frase. No es nuestro interés en este momento el análisis de la estructura de la cláusula, pero debemos detenernos en los argumentos subyacentes que pareceñ sustentar la nuclearidad del nombre.

En nuestra opinión, estos argumentos son de naturaleza semántica. Mo entran en juego características formales. La razón para considerar nuclear en una estructura de frase al sustantivo reside en la interpretación referencial del significado lingüistico. Y esta interpretación semántica del nombre hunde sus raíces en el análisis lógico de Aristóteles de sustancia y accidentes. Lo accidental será considerado opcional, a diferencia de la sustancia que se considera nuclear (González Escribano 1979-80:266). No pueden ser considerados nucleares los elementos que carecen de significado referencial.

No es necesario recordar las numerosas criticas que de esta interpretación del significado lingüístico como etiquetas de los objetos del mundo han realizado a lo largo del tiempo filósofos, lingüistas, antropólogos... Ya el propio Saussure advertía contra la confusión entre nombre y realidad. De igual modo, es frecuente en la semántica y pragmática actuales subrayar el carácter intencional e interpersonal de las lenguas naturales en contraposición al valor informativo, tradicionalmente considerado primordial del lenguaje 
humano. Baste citar la opinión de uno de los grandes lingüistas del siglo, L. Hjemslev, quien rechaza la distinción entre palabras "vacias" y "palabras llenas": "Las llamadas significaciones léxicas de ciertos signos no son sino significaciones contextuales artificialmente aisladas, o paráfrasis artificiales de las mismas. Totalmente aislado, ningún signo tiene significación; toda significación del signo surge en el contexto, entendiendo por tal un contexto situacional o un contexto explícito (...). No imaginemos, pues, que un sustantivo, por ejemplo, es más significativo que una preposición, o una palabra más que una terminación derivacional o flexional" (Hjemslev 1943:70).

Sin embargo, este principio del núcleo como elemento con significado léxico subyace en el análisis de la estructura de frase de la mayoría de las gramáticas, antiguas y modernas $^{4}$. Uno de los principios constitutivos de la teoría estándar de la estructura de frase teoria X-barra- es la lexicalidad, esto es, que todas las categorias frásticas son proyecciones de las categorías léxicas (Kornai-Pullum 1990:25). Del mismo modo, señala A. Siewierska $(1991: 10)^{5}$ que en la Gramática Funcional de S. Dik los núcleos son necesariamente elementos léxicos.

B) Pese a esta fundamentación semántica, desde el principio- vid. arriba Apolonio- se buscaron tests que determinaran el elemento nuclear. Esta prueba heurística es la sustitución: todo elemento que puede ser conmutado por cero en la cadena debe ser considerado opcional. Obviamente, esta prueba de la conmutación por cero sólo es válida para una gramática que se construya libre o independiente de contexto, pues de otro modo, en contexto apropiado, cualquier término- categona léxica o no- puede aparecer aisladamente por lo que seria considerado nuclear o bien, en otros análisis, con elemento nuclear no visible o vacío.

Pues bien, si aplicamos esta prueba de la conmutación por cero a una secuencia como la siguiente:

2. «(...) No me puedo permitir ese lujo (...)" (p.34).

Resultaria:

2a. No me puedo permitir lujo

2b. No me puedo permitir ese

La primera de las secuencias es claramente inaceptable, mientras que la segunda no lo es. Ciertamente, para la segunda pueden existir distintos análisis: considerar al demostra-

\footnotetext{
${ }^{4}$ De la persistencia de esta idea puede ser muestra la gramática tagmémica. Pese a dedicar esfuerzo y atención al concepto de núcleo, defienden- algunos autores- la existencia de elementos obligatorios no nucleares, esto es, distinguen elementos obligatorios, elementos que aparecen necesariamente en el sintagmema, y elementos obligatorios nucleares, siendo éstos últimos portadores de significado léxico (González Escribano 1979-80: 290).

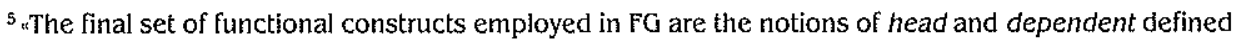
more or less as in standard dependency theory (see e. g. Hudson 1980). The only notable departure from standard treatments of these functions is that the head is necessarily lexical which is interpreted by Dik as excluding auxiliary verbs and adpositions from head status (...)" (Siewierska 1991: 10; el subrayado es nuestro).
} 
tivo elemento nuclear o bien considerar que continúa siendo determinante con núcleo elíptico o vacio. Trataremos más abajo sobre ambos análisis. Lo único que nos interesa notar por el momento es que la secuencia $2 \mathrm{~b}$ es aceptable, a diferencia de $2 \mathrm{a}$. $\mathrm{Y}$ ello sucede en cualquiera de las posiciones sintácticas- sujeto, objeto, predicado, suplemento, término .... y para cualquier tipo de nombre (contable o no contable).

Ejemplos:

a) En posición de sujeto:

3. (...) y cuando vi que aquellas grabaciones eran monstruosas..., (...) (p.299)

3a. Y cuando vi que grabaciones eran monstruosas

3b. Y cuando vi que aquellas eran monstruosas

b) Como suplemento:

4. O sea el Ateneo no ha hecho sino participar de, de esa... V... despolitización(...)(p. 108).

4a. O sea el Ateneo no ha hecho sino participar de despolitización

4b. O sea el Ateneo no ha hecho sino participar de esa

Ello no significa que no puedan hallarse ejemplos donde es posible suprimir el determinante, resultando secuencias aceptables:

5.(...) son mayores a los inconvenientes. Pero esos inconvenientes surgen.(p. 144)

5a. Pero inconvenientes surgen

$5 \mathrm{~b}$. Pero esos surgen

Si la prueba de la conmutación por cero que introduce Apolonio hubiera sido determinante para elegir el elemento nuclear de una secuencia, deberian haberse producido, al menos, serias dudas, para el español, respecto de la nuclearidad del nombre. Como decíamos más arriba, son las razones semánticas las que, en realidad, inclinan al análisis del determinante como elemento dependiente.

Como apoyos a este análisis se ha podido aducir la debilidad fonética de los determinantes típicos, el artículo y el posesivo. Ambos son clíticos y se apoyan fónicamente en el segmento que les sigue. Sin embargo, este argumento no es válido para todos los elementos de la clase, no para el demostrativo. Por otro lado, es incuestionable que el elemento que apoya fonéticamente al artículo o al posesivo no lo convierte en elemento nuclear de la frase, asi, p. ej, "la muy verdadera historia de S. Francisco". Del mismo modo, sería preciso demostrar que existe una relación constante e invariable entre tonicidad y nuclearidad sintáctica. En fin, el carácter clítico de algún determinante no es prueba sintáctica suficiente para determinar su carácter de complemento.

En suma, el análisis del nombre como núcleo de la frase sustantiva se apoya en ideas excesivamente generalizadoras: por un lado, en la consideración del nombre como sujeto 
y éste como elemento obligatorio de una oración y por otro, en la interpretación de la significación del nombre como significado referencial. De hecho, las caracteristicas morfológicas y de clítico de algunos determinantes permiten apoyar la tesis del núcleo como elemento dotado de significado léxico. De igual modo, el test de la conmutación por cero muestra que en numerosas ocasiones puede ser eliminado el nombre de la frase sin que por ello se produzca una secuencia inaceptable.

En realidad, se defiende que un elemento nuclear debe referir a un objeto real ${ }^{6}$, o en una versión más débil, al hecho de que sólo los elementos con significado léxico pueden desempeñar función nuclear. Esta es la idea subyacente básica y el que parece ser mayor obstáculo para considerar a un elemento morfológico elemento nuclear.

2.2. La segunda de las posiciones arriba expresadas opta por considerar que la relación entre el nombre y el determinante es de interđependencia, relación sintáctica binaria y por tanto, no nuclear, esto es, la construcción es exocéntrica.

2.2.1. De acuerdo a su definición, interdependencia es la "relación entre dos( o más) unidades en la que la presencia de cada una de ellas es necesaria para la presencia de la(s) otra(s)" (Rojo-Jiménez 1989: 45; el subrayado es nuestro). Este concepto, procedente de la escuela glosemática de L. Hjemslev, fue aplicado inicialmente por G. Rojo a la tipología oracional, pero recientemente se ha ampliado su uso a relaciones entre otros tipos de unidades. Sin embargo, como en el caso de las oraciones, es dificil determinar si la exigencia mutua a la que se refiere la definición es de naturaleza pragmática, sintáctica o semántica. Si se tratara de exigencia semántica, probablemente habría que afirmar que la relación que, en algunos casos, se establece entre un núcleo predicativo y su complemento directo subcategorizado es de interdependencia. No parece que sea éste el caso, dado que afirman que existe relación de determinación o subordinación entre el predicado y el complemento directo (R-J: 46).

Dejando a un lado estas observaciones teóricas, G. Rojo y T. Jiménez para determinar la relación existente entre los elementos constitutivos de una frase nominal la someten a la ya conocida prueba de la conmutación por cero. De este modo resulta:

"(61a) La leche es un buen alimento

$(61 \mathrm{~b})^{*}$ Leche es un buen alimento" (R-J: 124)

Con este y otros ejemplos pretenden demostrar que la relación es de interdependencia, puesto que tampoco es posible " ${ }^{*}$ La es un buen alimento". Por ello se distingue entre una "frase sustantiva", frase cuyo núcleo es un nombre sin determinante, normalmente en plural, y una "frase nominal", frase sin núcleo en la que se distinguen dos nuevas funciones: "determinante" y "nominal".

Como hemos visto más arriba, la conmutación por cero es prueba únicamente válida para una gramática indepenđiente de contexto, y por otro lado, estos resultados inacepta-

\footnotetext{
${ }^{6}$ Muy cercano a esta posición está A. Zwicky. Define el argumento semántico: «(...) in a combination $X+Y, X$ is the 'semantic head' if, speaking crudely, $X+Y$ describes a kind of thing described by $X "$ (Zwicky 1985:4; subrayado mio).
} 
bles suceden únicamente cuando el determinante que se estudia es el artículo $u$ otro clítico. La razón para que esto suceda no nos parece de carácter sintáctico, sino simplemente fónico. Si reemplazamos en el ejemplo de G. Rojo la forma clitica por la forma tónica correspondiente del articulo, esto es, "la" por "ella", el resultado es similar al de los demostrativos, determinantes tónicos.

Incluso si tomamos por ejemplo- ejemplo ad hoc ciertamente, pero secuencia posibleel enunciado: "La niña ama a su madre", y eliminamos el sustantivo "niña", la secuencia resultante es aceptable: "La ama a su madre". Evidentemente nos encontramos con relaciones funcionales diferentes, pero simplemente queremos mostrar que si un elemento clitico encuentra apoyo fonético la secuencia puede interpretarse, en determinadas condiciones. Las diferentes relaciones funcionales se producen porque el articulo es clítico que se agrupa fónicamente en contextos distribucionales, generalmente, nominales, a diferencia del pronombre personal átono con distribuciones verbales.

De nuevo, el entender como determinante prototípico un elemento átono, el artículo, ha conducido a una conclusión no satisfactoria, al menos no para toda la clase.

2.2.2. Del mismo modo, la construcción de las frases nominales es caracterizada como exocéntrica. Las construcciones exocéntricas son, por definición, "construcciones en las que sólo puede haber dos constituyentes al nivel más alto y esos dos elementos se exigen mutuamente, de tal forma que ninguno de ellos aisladamente es capaz de desempeñar la función que realiza el conjunton (R-J: 122). Pese a que la relación de interdependencia- vid. más arriba- ni la construcción exocéntrica ${ }^{7}$ exigen, según sus definiciones, agrupaciones binarias, en la definición arriba citada se hacen corresponder conceptos como los de interdependencia, exocentrismo y agrupación binaria.

Con respecto al binarismo sintáctico, que es rasgo determinante de las construcciones interdependientes- frase nominal, frase preposicional, suplemento, y caracteristico de las oraciones frente a las cláusulas- y por consiguiente, rasgo altamente caracterizador y productivo para un buen número de construcciones sintácticas de distintos niveles y no sólo las denominadas exocéntricas, parece que debería motivarles el reconsiderar la inicial caracterización del análisis sintáctico como no binarista (R-J: 23). Es posible que las ramificaciones de tres constituyentes pudieran reducirse a ramificación binaria, y por tanto, no faltarian etiquetas (R-J: 24), si se considerara la existencia del nivel intermedio entre la frase, proyección máxima, y la categoria, preterminal. Nos referimos al nivel de una barra de la teoría generativista.

De otro lado, presentaría dificultades a este análisis binarista, exocéntrico e interdependiente la combinación de un determinante y cuantificador precediendo a sustantivo o con ausencia de éste. En una secuencia como:

6. "Éramos los dos alumnos del mismo profesor; se llamaba, recuerdo, Adolfo,(...)" (p.9; subrayado nuestro).

\footnotetext{
${ }^{7}$ Más arriba se define la construcción exocéntrica sin hacer referencia a su carácter binario. Sólo se opone a la construcción endocéntrica que posee un elemento, el núcleo, capaz de desempeñar la función de toda la construcción: "En las construcciones exocéntricas, en cambio, no puede haber núcleo, ya que, por definición, ninguno de sus constituyentes inmediatos puede desempeñar la función de toda la construcción" (Rojo-Jiménez: 105).
} 
6a. Éramos dos alumnos del mismo profesor...

6 b. Éramos los alumnos del mismo profesor...

Los dos elementos subrayados de la oración pueden ser sustituidos alternativamente $\sin$ que se produzca una frase inaceptable ( 6 a y $6 \mathrm{~b}$ ), aunque no sean semánticamente equivalentes por la pérdida de información. A la vista de este comportamiento la relación binaria que para la "frase nominal" se defiende no puede ser mantenida, salvo que se considerara que la función "determinante" puede soportar "modificaciones", pero en ese caso seria igualmente de dificil determinación cúal de los elementos es el elemento adjunto o complemento y cuál de ellos el núcleo. Incluso puede ser eliminado el sustantivo núcleo de la función "nominal" sin que se advierta inaceptabilidad.

En cualquier caso, la combinación de cuantificadores y determinantes ofrece numerosas dificultades para cualquier propuesta de análisis. Nuestra intención era mostrar una de las dificultades con la que tropieza el análisis de la frase nominal exocéntrica que defienden G. Rojo y T. Jiménez.

2.2.3. Respecto del exocentrismo y la ausencia de núcleos y como consecuencia, de relaciones de determinación, parece solución demasiado costosa y escasamente productiva en el análisis sintáctico. No se observan aparentemente ventajas.

Dejando a un lado, por encontrarse ya en los límites del análisis sintáctico, la caracterización de las oraciones- en el sentido de G. Rojo-como construcciones exocéntricas, nos parece posible que las unidades de nivel de frase caracterizadas como exocéntricas podrian ser consideradas endocéntricas. Dado que el suplemento es una frase preposicional, bastaría con postular un análisis endocéntrico de la frase preposicional, en el que no podemos detenernos, y de la frase nominal.

Esta solución presenta como ventaja la eliminación de una serie de funciones que serian no generalizables y puramente idiosincráticas: "determinante" y "nominal", "término" $\mathrm{y}$ "director".

El caso de la función "suplemento" es algo particular. El análisis de G. Rojo y T. Jiménez hace hincapié en las relaciones intrafrásticas. Se pone de relieve la interdependencia de sus constituyentes, hecho innecesario, puesto que esta función se realiza por una frase preposicional, prototipo de relación interdependiente en su análisis. Es probable, y por ello, su insistencia, que se esté pensando en una relación diferente asimismo entre "predicado" y "suplemento". Si así fuera, y a ello se añade el hecho de que la preposición también podría agruparse con el lexema verbal, seria necesario dar otra etiqueta incluso a la función "predicadon. La relación entre "Suplemento" y "predicado" es de naturaleza diferente a la de "predicado" y "complemento directo", una relación de determinación. Quizá seria, pues, necesario, si son atinadas estas conjeturas, modificar la etiqueta funcional no sólo del complemento verbal, el "suplemento", sino también del "predicado".

Para el caso de la frase nominal no creemos que existan mayores obstáculos en considerar que el determinante ejerce la función de núcleo de la frase sino la inercia de la tradición y la relación vinculante entre núcleo sintáctico y significado léxico. Manifiestamente, G.Rojo y T. Jiménez advierten del carácter técnico del concepto de núcleo y no reducible a "undamental", o "Sustancial" (R-J: 105). El concepto de núcleo es sinónimo de elemento obligatorio frente al del complemento o modificador que se considera opcional (R-J: 118, 123-4). 
De acuerdo con esto, en una secuencia como:

7. (...)si, el piso pequeñito, con una habitación de ésas que sirve de todo ¿no? de salón, de cuarto de estar (p.72; subrayamos nosotros).

El término subrayado no aparece junto al que en este análisis se denomina "nominal", luego será imposible defender que existe relación de interdependencia y exocentricidad en esta frase. La solución más común para secuencias similares a ésta ha sido postular la elipsis del elemento estimado como nuclear, dado el análisis del sustantivo como elemento nuclear de la frase. Sin embargo, debe observarse que el antecedente textual que posibilita la recuperación del elemento presuntamente elidido no coincide en número: "habitación / ésa-s" en este caso, y que en otros muchos no es posible recuperar ningún "nominal".

Por otro lado, como afirma T. Jiménez(1991:233), el elemento recuperado uno implica la necesidad de suponer que estos elementos son realmente 'parte de la estructura' de estos sintagmas". No obstante, los casos de "falsa elipsis" que estudia T. Jiménez en este trabajo corresponden a las secuencias: art. + frase prep., art. + adj. y art. + orac. relativo, que analiza, tal como ya se hacia en G.Rojo -T.Jiménez (1989:125), sin recurrir a elipsis, como frase exocéntrica con nominal no sustantivo, sino nominal constituido por frase (preposicional o adjetiva), palabra(adjetivo) o cláusula. Probablemente, si se hubieran usado otros determinantes, o bien se aceptara la distribución complementaria entre artículo y pronombre personal de tercera persona, se habria reparado en que no es necesaria la aparición del "nominal", y por ello no es posible defender la exocentricidad ni interdependencia.

Por tanto, si los ejemplos usados no contienen términos cliticos, el comportamiento sintáctico del determinante parece similar al de cualquier otro núcleo.

2.3. La tercera de las opciones, a saber, que existe una relación de dependencia, pero que, a diferencia de la primera, es el determinante el elemento subordinante y el sustantivo o cualquier otro elemento que le siga el elemento dependiente cuenta asimismo con una larga nómina de defensores. Mos fijaremos en las más recientes aportaciones.

Recordemos (vid.2.1) que uno de los argumentos que podian manejarse para defender la nuclearidad del sustantivo en una frase nominal derivaba del análisis de nombre y verbo, de sujeto y predicado, como los dos términos y funciones capaces de constituir por sí solos oración completa, y que, asimismo, esta nuclearidad funcional había inspirado incluso la disposición material de los capitulos de numerosas gramáticas.

Pues bien, justamente cuando en el análisis sintáctico se produce un cambio en la concepción de los elementos constitutivos y nucleares de la oración aparecerá la defensa de la interpretación del determinante como núcleo de la frase. Así sucede en la teoria de frase generativista ${ }^{8}$. $N$. Chomsky postula la necesidad de considerar nuclear el elemento INFL (FLEXión verbal) en la oración, y poco después aparecen propuestas en favor de considerar DET(erminante), como núcleo de la hasta entonces denominada frase nominal

\footnotetext{
${ }^{8}$ El trabajo considerado fundador de la teoria X-barra "Remarks on Nominalization" (1970) de Chomsky trata también sobre las relaciones entre la frase nominal y la estructura oracional.
} 
y a partir de entonces, frase determinante. Basta considerar el titulo de la tesis de S. Abney (1987: The English Youn Phrase in its Sentential Aspect), uno de los defensores de la frase determinante, para reparar en ello.

No obstante esta vinculación no es asumida por todos los generativistas. Hay autores que aceptan el nudo FLEX, pero no el análisis de la frase determinante (Haegemann 1991:89 n.11). Defienden un análisis similar al tipo A, a saber, el determinante ocupa la posición de Especificador de la frase nominal, cuyo núcleo es el sustantivo.

Del análisis de DET sólo retendremos la argumentación central que lo justifica. No podemos detenernos en las numerosas consecuencias internas a la teoría, para la teoria de la estructura de frase y otros principios, que este análisis conlleva. El análisis de la frase determinante se sustenta en la consideración de un tipo de categoria distinta a la categoria léxica, la categoria funcional.

Las categorias léxicas de la teoría de la estructura de frase: nombre, verbo, adjetivo y preposición, que, como puede observarse por la inclusión de la preposición no coinciden exactamente con los elementos de significado léxico, tenían como característica principal la de ser predicados, esto es, la de tener una red temática. A diferencia de ellas las categorías menores o no léxicas no poseían esta propiedad y no funcionaban como núcleos de frase. Eran categorías que no jugaban un gran papel en la teoria de la frase. Sin embargo, como deciamos más arriba, la propuesta de frases -IP, CP- cuyos núcleos no eran categorias léxicas provocó una revisión de esta división inicial de categorias. Las categorías que hasta entonces recibían la caracterización genérica de no léxicas pasaron a denominarse funcionales.

Las categorias funcionales se caracterizan, según S. Abney (1987) y N. Fukui \& M. Speas (1986), por: a) ser clases cerradas; b) tener un solo complemento, que no es usualmente un argumento; $\mathrm{c}$ ) generalmente, ser dependientes morfológica y fonéticamente (clíticos, a menudo; o elementos nulos); d) no poder ser separados de su complemento; e) no tener significado descriptivo; f) tener un solo especificador, a diferencia de las categorías léxicas; g) ser movidos los especificadores de núcleos funcionales desde su complemento; h) tener todos los núcleos funcionales posición de especificador, dado que no está claro que todos los núcleos léxicos tengan posición de especificadores; e i) las lenguas a las que le faltan núcleos funcionales también les faltan posiciones de especificador.

Las características a), c) y e) corresponden a las que normalmente se han asignado a los morfemas. Las restantes características se refieren a las consecuencias internas que, para la teoria de la estructura de frase y para la tipologia de lenguas- rasgo i)-, se derivan de este nuevo tipo de categorias sintácticas: la asimetria de la estructura de frase, la hipótesis de un "no-uniforme" nivel de barras. No nos detendremos en ello. Pensamos que no afecta a los propósitos que perseguimos, a diferencia de los rasgos a), c) y e).

Según esta caracterización, las categorias funcionales son morfemas, pero morfemas que tienen un comportamiento particular. De nuevo, del análisis de las relaciones entre sujeto y predicado se deduce este comportamiento. Las caracteristicas morfológicas del verbo- concordancia y flexión temporal-modal- asignan caso nominativo en el nivel $\mathrm{V}^{\prime}$, que ocupará la plaza del especificador de la $\mathrm{FF}$, la frase flexionada, esto es, la oración. De este modo, aunque no tienen red temática, las categorías funcionales asignan caso a la izquierda, y no a la derecha, o mejor, kaso (Fukui-Speas 1986: 138). Con este término se hace referencia a los casos de las categorias léxicas y a los rasgos funcionales asignados por las categorías funcionales. 
En la frase determinante, sin embargo, no siempre se asigna kaso, y por tanto, no siempre es ocupada la posición de especificador. En inglés sólo "'s ", en distribución complementaria con el articulo, asigna caso genitivo: "John's book".

En fin, a determinadas características morfológicas, relacionadas normalmente con la concordancia nominal y verbal, se les concede en la teoria generativista el papel de núcleo de la frase. La inicial asimetría entre categorias léxicas y no léxicas o funcionales, que se apoyaba en que existen categorias que pueden tomar argumentos y categorías que no pueden, se mantiene, dado que el kaso de las categorias funcionales es posición no argumental y no temática, pero, no obstante, se defiende que existe una red kaso, que incluye al caso y a los rasgos funcionales, rasgos-f.

Queremos hacer hincapié en la recurrencia como rasgo discriminador de categorias léxicas y funcionales, del significado descriptivo, que normalmente va acompañado de red temática-la preposición, posible excepción, es, para algunos, categoria fronteriza o plenamente funcional (Abney)-. Una vez más, se recupera la vieja concepción del significado como referencia e incluso se afirma que los núcleos funcionales son "parásitos semánticos":

The difference between lexical and functional heads is that the position in the Kase grid of a functional head is never linked to a variable in an LCS (Estructura léxicoconceptual]. The reason is that there are no referential variables in the LCS of a functional head. A functional head is in an informal sense semantically parasitic on a predication, and so although it has Kase features to assign, it has no relevant variable in its LCS to which these might be linked (Speas 1990:116; subrayado nuestro).

En suma, la distinción entre categorias léxicas y funcionales es de naturaleza esencialmente semántica y la nuclearidad sintáctica de las categorias funcionales parece apoyarse, no exclusivamente, en la concordancia morfológica del núcleo y complemento.

3. El argumento semántico es, pues, el más comúnmente usado para o bien defender la nuclearidad del nombre o bien la adscripción del determinante a las categorías funcionales. Pues bien, este argumento, como ya vimos, se apoya en la errónea identificación de significado y referente o cosa. No es necesario argumentar largamente sobre esta cuestión: una cosa puede ser designada mediante diversos significados y el significado puede no referir a cosa alguna.

3.1. Sin embargo, desde un punto de vista semántico, puede defenderse (Trujillo 1988: 144 y ss.; 237 y ss.) que la función semántica "determinante" es la propiedad semántica "necesaria" de un sintagma nominal, esto es, los sustantivos y adjetivos son semánticamente dependientes: "La mención determinativa puede ser totalmente autónoma, sin que sea necesario subentender contenidos léxicos en todo caso(...) "(Trujillo 1988:149). La extraneza que pudiera producir este análisis se debe al arraigo de la tradición, que interpretaba al sustantivo como la clase de palabra que designa cosas, esto es, a la vinculación de significado y cosa, y por otro lado, se debe a la confusión de interpretación y comprensión de una secuencia lingüística. Identificar un referente, dar la interpretación de un enunciado y asignarle sentido es un proceso discursivo, textual en el que entran en juego diversos mecanismos inferenciales e información no exclusivamente lingüistica. Por el contrario, "Comprender" el significado de una secuencia es una operación de lengua que no consiste 
en "buscarle un equivalente en el mundo(...), sino reconocerlo como realmente es en si mismo, haciendo abstracción de la realidad" (Trujillo 1988: 59).

De este modo, dado que se ha mostrado que el principal argumento que apoyaba el análisis del sustantivo como núcleo de la frase nominal o sustantiva era erróneo y que es asumible postular la existencia de una función semántica determinante en la frase, el hasta ahora denominado "sintagma nominal" puede considerarse frase determinante.

La frase determinante es, pues, frase de naturaleza endocéntrica caracterizada por la presencia del determinante, elemento obligatorio y equivalente distribucional del constructo, salvo para determinantes átonos.

Quizás podrían plantearse algunas dificultades de base morfológica a este análisis. Las relaciones de concordancia, género y número, entre los elementos de la frase determinante se han analizado normalmente considerando al sustantivo el determinante morfológico de la secuencia. Sin embargo, no se ha ocultado que, en ocasiones, es el determinante el portador del género y número de la frase. Nos referimos a los casos en que no existe en el sustantivo variación de número, ej. la/las crisis, o genérica, ej. el/la policia. La razón para mantener la consideración del sustantivo como determinante morfológico, pese a las pruebas empiricas contrarias, son, en nuestra opinión, de naturaleza semántica: la confusión de género y número- formas lingüisticas- con sexo y cantidad, conceptos de lo real.

Por otro lado, los determinantes cuentan incluso con una forma genérica de la que carecen los sustantivos: el neutro. Por ello, y al menos para el caso de los neutros, no podría afirmarse que el sustantivo exige o determina la forma morfológica del determinante. Por consiguiente, existen comportamientos y pruebas que permiten apoyar la idea del determinante, núcleo semántico y sintáctico, como lugar morfológico o núcleo.

\subsection{Este análisis del determinante como núcleo asimismo ofrece ventajas explicativas.}

3.2.1. La tradicional división de los determinantes como adjetivos determinativos y pronombres, según su función adjetiva o nominal, puede ser reemplazada por una visión de clase única, dado su comportamiento sintáctico relativamente homogéneo. Gracias a este análisis, podemos eliminar una duplicidad de etiquetas para elementos que poseian idénticas formas y, de acuerdo a este análisis, funciones homogéneas.

Ciertamente, en la clase de los determinantes existen formas en distribución complementaria- A. Bello o R. Trujillo 1988:147-, de acuerdo a su carácter átono o tónico: las del artículo y las del pronombre personal, pero su función y significado permanecen constantes, pese a esta variación formal.

Estas variaciones formales no justificarian separar en dos clases a estos elementos, del mismo modo que no se propondria una doble categorización para las formas "buen / bueno", formas diversas del adjetivo, pero que manifiestan identidad funcional y semántica en ambos usos.

3.2.2. Tanto en el caso de la aparición con sustantivo como con adjetivo, frase preposicional u oración de relativo, casos analizados normalmente como elípticos, la función que desempeña el determinante es la de núcleo. Defender que en los casos de determinante+ adjetivo, frase preposicional $u$ oración de relativo el sustantivo, núcleo de la frase, se ha elidido, tropieza con varias dificultades.

En primer lugar, el hecho de que el elemento que se analiza como obligatorio no esté 
presente en la cadena, prueba de carácter empírico. Normalmente se opta por postular que el elemento nuclear $u$ obligatorio debe considerarse en otro nivel distinto al de la superficie, al de los hechos directamente observables. Recurrir a un nivel de abstracción superior, pese a las pruebas empíricas contrarias, no parece necesario.

En segundo lugar, tropieza con la comprensión habitual de los fenómenos elipticos: la elipsis como fenómeno sintáctico sólo afecta a los elementos obligatorios y si esto es así, cómo puede explicarse que un elemento considerado en este análisis opcional, el determinante, sea de presencia obligatoria en la secuencia elíptica: "Todo ello permite afirmar que la característica estructural distintiva de las construcciones de SN elíptico es la obligatoria aparición del determinante ( y no la del determinante y el complemento, simultáneamente)" (Hernanz-Brucart 1987:197, subrayado nuestro).

Mo parecen existir razones que permitan apoyar este análisis. Si los procedimientos de "Sustantivación" tienen el inconveniente de la recategorización de unidades, el análisis del sustantivo elíptico parece analizar dualmente al determinante, elemento inicialmente opcional, como obligatorio en secuencias elipticas.

La argumentación que parece defenderse puede invertirse. De la consideración del sustantivo como núcleo en frases en las que éste aparece en la cadena se deduce su nuclearidad profunda o abstracta en secuencias en las que no aparece; pero, si se reconoce que el determinante es elemento obligatorio y, por tanto, nuclear en secuencias en las que no aparece el sustantivo, ¿qué impide considerar que es asimismo nuclear en secuencias en las que se acompana de sustantivo?

A ello debe unirse las dificultades que para la explicación sintáctica conlleva recurrir al concepto de elipsis. La elipsis y los procedimientos de recuperación de información no explicita son fenómenos habituales, sin duđa, en la interpretación de los enunciados. Sin embargo, explicar de qué modo y con qué medios se recupera información para conseguir la interpretación de los enunciados son tareas que salen fuera del marco de la Sintaxis y exceden su potencialidad explicativa. Son tareas propias de la Pragmática.

Y por último, las dificultades que plantea la caracterización de la posible categoria vacía: pro, PRO o variable... debería conducir, dado que su rendimiento explicativo es idéntico a la propuesta contraria: el determinante sin nominal nulo, a considerar no necesarios los nominales vacios, tal como defiende L. Eguren ${ }^{9}$ (1989).

3.3. Existen, no obstante, varias dificultades para este análisis: el distinto comportamiento de articulo y posesivos en frases "elípticas", las posiciones postnominales de posesivo y demostrativo... Sólo expondremos unas breves observaciones sobre una de ellas, el sustantivo que aparece sin determinante.

Es bien conocido que existen frases y posiciones sintácticas en las que el sustantivo puede aparecer sin determinante (Lapesa 1974). Algunos defensores de la hipótesis de la frase determinante han defendido que en estos casos el determinante es elemento vacio o elíptico (Abney 1987). Sin embargo, parece claro que si hemos rechazado más arriba la propuesta del sustantivo elíptico, no deberíamos aceptar, sin justificación razonada, la propuesta del determinante vacio.

Sabemos que los sustantivos que pueden aparecer sin determinante son los continuos

${ }^{9}$ Lamentablemente, no nos ha sido posible consultar la tesis de L. Eguren (1988). 
en singular o plural o bien los discontinuos en plural, y los nombres propios. En estos casos, el determinante parece elemento opcional, y por ello el sustantivo sería elemento obligatorio y nuclear. Una de las soluciones posibles es la que adoptan G. Rojo y T. Jiménez: existen frases nominales y frase sustantivas, en nuestro análisis frases determinantes y frases sustantivas. Sin embargo, parece que en, al menos, uno de los casos es posible defender la frase determinante. En una secuencia como:

8. ¿Le parece a usted que los medios de comunicación con las masas(...) tienden a suprimir estas diferencias o...(...) p. 38

8a. ¿ Le parece a usted que los medios(...)tienden a suprimir estas ...

8b. ¿ Le parece a usted que los medios(...)tienden a suprimir diferencias ...

Las tres frases son posibles. La segunda de ellas (8a), que puede parecer extraña, es tan comprensible como cualquiera de las otras dos. La dificultad no reside en su comprensión sino en su interpretación, como sucede también en (8a), el lector que no dispone de un contexto más amplio no puede interpretar, asignar referente a "estas diferencias". Por otro lado, (8b) muestra que es posible la desaparición del determinante, pero, si la sometemos a una prueba más, resulta:

8c. ¿ Le parece a usted que los medios(...)tienden a suprimir diferencia ...

(8c) no es aceptable. La única diferencia entre (8b) y (8c) residen en la diferencia de número, por lo que no puede afirmarse para estos casos que el elemento obligatorio es el sustantivo, que permanece en (8c), sino el número plural. Por tanto, los determinantes morfológicos pueden considerarse frases determinantes. Su elemento nuclear, a diferencia de otras frases determinantes, son elementos trabados, no discretos. Esto es, la función nuclear de una frase determinante puede ser llenada por elementos discretos o trabados.

Para el caso de los nombres continuos y propios, pese a que son igualmente frases con determinación, léxica en estos casos, parece plausible, dado que no existen elementos trabados o discretos que llenen esta función, conceder que se trata de frases sustantivas. No obstante, esta solución debe considerarse provisional. Es necesario dar explicación, entre otros fenómenos, a la curiosa posposición en la que suele aparecer el nombre continuo sin determinante en función de sujeto, ej. "sale agua del grifo" o a la necesidad de que aparezca el determinante cuando el nombre propio se acompaña de adjetivos o frases restrictivas: ej. "El Madrid de postguerra".

4. Queremos insistir en la limitación de este trabajo. Han quedado fuera o relativamente marginados problemas sintácticos de indudable interés: la posición pre y postnominal de demostrativos, las condiciones en las que aparece una frase sustantiva, las relaciones entre los determinantes y los cuantificadores, determinantes y pronombres...

Nuestro propósito era bien modesto: exponer algunas dificultades con las que tropieza el concepto de núcleo y mostrar la, a veces explícita y en ocasiones subyacente, posición que vincula significado léxico y nuclearidad sintáctica. 


\section{REFERENCIAS}

Abney, S. (1987): The English Noun Phrase in its Sentential Aspect. Tesis doctoral. MIT.

Apolonio Discolo (1987): Sintaxis. Introducción, traducción y notas por Vicente Bécares Botas. Madrid: Gredos

Bello, A. $\left(1860^{5}\right)$ : Gramática castellana destinada al uso de los americanos. Con las Notas de Rufino José Cuervo. Estudio y edición de Ramón Trujillo. Madrid: Arco/Libros, 1988

Eguren Gutiérrez, L. (1988): Sintaxis de la frase nominal: la hipótesis de la frase determinante. Tesis doctoral inédita. Valladolid: Universidad de Valladolid (1989): V Congreso de Lenguajes Maturales y Lenguajes Formales. Barcelona: Universidad de Barcelona, 481-492

Esgueva, M. y M. Cantarero (1981): El habla de la ciudad de Madrid. Materiales para su estudio. Madrid; CSIC.

Fukui, N. and M. Speas (1986): Specifiers and Projections. MIT Working Papers on Linguistics 8, 128-172

González Escribano, J.L. (1979-80): Reflexiones acerca del concepto de "Núcleo" en la gramática tagmémica. Archivum XXIX-XXX, 265-310

Haegeman, L. (1991): Introduction to Government and Binding Theory. Oxford: Blackwell

Hernanz, M‥Ll. y J.Mㄹ. Brucart (1987): La sintaxis. 1. Principios teóricos. La oración simple. Barcelona: Critica

Hjelmslev, L. (1943): Prolegómenos a una teoría del lenguaje. Madrid: Gredos, 1984

Hudson, R. (1987): Zwicky on Heads. Journal of Linguistics 23, 109-132

Huddleston, R. (1984): Introduction to the Grammar of English. Cambridge: Cambridge University Press, 1988

Jiménez Juliá, T. (1991): Elipsis nominal y no realización en español. En M. Brea e F. Fernández Rei (coords.) (1991): Homenaxe ó Profesor Constantino Garcia. Vol.l. Santiago: Universidad de Santiago, 223-241

Kornai, A. and G.K. Pullum (1990): The X-bar theory of phrase structure. Language, 66, 1, 24-50

Lapesa, R. (1974): El sustantivo sin actualizador en español. En R. Lapesa (1987): Estudios lingüisticos, literarios y estilisticos. València: Universitat de València, 57-68

Mebrija. A. de (1992)(1492): Gramática de la lengua castellana. Edición critica de Antonio Quilis. Madrid: Ediciones de Cultura Hispánica

Rojo, G. y T. Jiménez Juliá (1989): Fundamentos de análisis sintáctico funcional. Santiago: Universidad de Santiago

Rothstein, S. D. (1989): Heads, Projections, and Category Determination. En K. Leffel and 
D. Bouchard (eds.) (1989): Views on Phrase Structure. Papers presented at a conference held at the University of Florida, Gainesville, in March 1989. Dordrecht: Kluwer, 1991, $97-112$

Siewierska, A. (1991): Functional grammar. London: Routledge

Speas, M.J. (1990): Phrase Structure in Natural Language. Dordrecht: Kluwer.

Sperber, D. and D. Wilson (1986): Relevance. Oxford: Blackwell

Trujillo, R. (1988): Introducción a la semántica española. Madrid: Arco Libros

Zwicky, A. (1985): Heads. Journal of Linguistics 21, 1-29. 